\title{
A245＼cjkstart重なり粒子画像における粒子数の計測方法
}

\author{
山本富士夫(福井大) 阮 暁東 (中国浙江大学) \\ 宋 向群 $\left(\right.$ 福井大 ${ }^{\circ}$ 井口 学(北海道大学大学院)
}

\section{A method for Counting Particle Number in Overlapped Particle Images}

\author{
Fujio YAMAMOTO*, Xiao-Dong RUAN**, \\ Xiang-Qun SONG*, Manabu IGUCHI ${ }^{* * *}$
}

\begin{abstract}
This paper describes a method to separate and count the number of particles in an overlapped image. The edges in an image are detected by Canny's method, and contours are linked from the pixels in edges. Contours are segmented using a criterion based on the derivative of the curvature. Constant curvature segments are clustered according to the relations among contour segments which are likely to represent the same circles. A minimization criterion is used to find out the circle parameters that provide accurate parameter estimation. The method can recover many circles in a heavily overlapped particle image, and provide a useful way in some practical applications, such as counting particles, separating overlapped bubbles in PIV system.
\end{abstract}

\section{Introduction}

The problem of circular contour recognition has received much attention in the field of pattern recognition and computer vision, because a segmented curve can provide us with a meaningful and compact form to analyze many round objects. In the earlier researches, the number of round objects in an image was limited and their sizes were almost the same. However, for some images, such as an image of coal ash, bubble plume, the traditional methods can not provide us with a satisfied separating result. The classical approach of analyzing the pattern is Hough transformation (Duda \& Hart, 1972). Although it is very simple, it needs heavy computational time and memory. Another problem is edge detection. The traditional approach to find out the edges was binarization method, i.e., searching the boundary of colonies by a binarization method (Sagaue, 1983). This method can only deal with the images with a few round objects, if the number of round objects is very large (see Figure 1), most information of edges will be lost due to the procedure of binarization.

Recently a number of papers reported the new development of the recognition of circular contour and the estimation of parameters of a circle. In the paper of Pla (1996), the procedure which could obtain accurate tangent values was described. Chen (1995) proposed an approach to segmentation of curves into arcs and line segments. Thomas (1989) proposed a simple approach for estimation of two parameters of a curve, i.e., circular centers and radii. In the paper of Lim (1995), the estimation of a circle was also presented. On the other hand, among many approaches to the edge detection, Canny's method (Canny, 1986; Castelow, 1988) proved to be an effective way to detect the edges in an image. In this paper, we used Canny's method to detect the edges, contours are segmented using a criterion based on the derivative of the curvature, which was originally reported by Pla (1996).

\section{Edge detection}

In order to detect the edge in an image, Canny's method is used in the present research. A two-dimensional Gaussian $\boldsymbol{G}$ in an estimated direction $\boldsymbol{n}$ which is oriented normal to the direction of and edge are defined as:

$$
\boldsymbol{G}=\exp \left(-\frac{x^{2}+y^{2}}{2 \sigma^{2}}\right), \quad \boldsymbol{n}=\frac{\nabla(\boldsymbol{G} * I)}{|\nabla(\boldsymbol{G} * I)|}
$$

* Dept. of Mech. Eng., Fukui University, 3-9-1 Bunkyo, Fukui, 910, Japan

** State Key Laboratory of Fluid Power Transmission and Control, Zhejiang University, 310027, China

*** Graduate School of Hokkaido University, Kita-ku, Sapporo, 060, Japan 
where $I$ is an image, and * denotes convolution. The operator is:

$$
\frac{\partial^{2}}{\partial \boldsymbol{n}^{2}}(\boldsymbol{G} * I)=0
$$

We firstly convolute an image with a symmetric Gaussian $G$ and then the location of edge is found so that the value of the directional second derivative takes zero according to Eq.(2), edge's strength is the magnitude of $|\nabla(G * I)|$.

\section{Contour segmentation}

After applying Canny operator to the image, we can detect edges with one pixel width. Linking these pixels in edges, we can obtain the curve segments. In this paper, the curvature function is used to characterize curve points, because the same segment points with the same constant curvature value means that these points in the segment are likely to belong to a single circle.

It is important to calculate the curvature functions along the curve accurately because the parameters of a circle are estimated by them. Let $\alpha(s)=(x(s), y(s))$ represent a regular curve where $s \in[0, L]$ is measured along the curve. Denote $\theta(s)$ as the angle defined by the tangent at the point $\alpha(s)$. The curvature $k(s)$ of $\alpha(s)$ is defined as $k(s)=d Q(s) / d s$. The tangent at a point $\left(x_{0}, y_{0}\right)$ can be approximated by the chord defined by two points of the curve $\left(x_{L}, y_{L}\right)$ and $\left(x_{R}, y_{R}\right)$, one to either side of $\left(x_{0}, y_{0}\right)$, where both points are as close as possible to $\left(x_{0}, y_{0}\right)$. The angle $\theta$ of the tangent at $\left(x_{0}, y_{0}\right)$ is

$$
\theta=\tan ^{-1}\left(\frac{y_{R}-y_{L}}{x_{R}-x_{L}}\right)
$$

In order to reduce the sampling error, instead of taking $\left(x_{L}, y_{L}\right)$ and $\left(x_{R}, y_{R}\right)$ as points of the curve, the coordinates of these points are calculated by averaging the coordinates of a group of neighbors to reduce the effect of noise.

\section{Clustering algorithm}

Once the curvature along the curve is obtained, the next step is to perform some processes, such as interpolation, smoothing and separating a curve into several arcs with the same constant curvature. The final step is grouping segments to find the relations between segments from any part of a contour or even between different contours. The clustering algorithm is applied to find out circles.

We start from the initial set of segments. Initially each segment is considered as the only element of its own group. An iteration procedure is performed, and then two groups of segments are considered as one segment if their distance function is small enough. The iteration is carried out until the same segments are not found.

\section{Parameter estimation}

Given a set of coordinates $\left(x_{1}, y_{1}\right),\left(x_{2}, y_{2}\right), \ldots,\left(x_{N}, y_{N}\right)$, a circle with center $\left(x_{c}, y_{c}\right)$ and radius $R$ is defined. $J$ is defined as

$$
J\left(R, x_{c}, y_{c}\right)=\sum_{i=1}^{N}\left(R^{2}-\left(x_{i}-x_{c}\right)^{2}-\left(y_{i}-y_{c}\right)^{2}\right)^{2}
$$

The function $J$ should be minimized with respect to $R, x_{\mathrm{c}}$ and $y_{\mathrm{c}}$. Differentiating Eq. (4) with respect to $R, x_{\mathrm{c}}$ and $y_{\mathrm{c}}$, respectively, we can solve these differentiation equations for $x_{c}$ and $y_{c}$. The final results are:

$$
\begin{aligned}
& a_{1}=2\left(\sum^{2} x_{i}-N \sum x_{i}^{2}\right) \\
& a_{2}=b_{1}=2\left(\sum x_{i} \sum y_{i}-N \sum x_{i} y_{i}\right) \\
& b_{2}=2\left(\sum^{2} y_{i}-N \sum y_{i}^{2}\right) \\
& c_{1}=\sum x_{i}^{2} \sum x_{i}-N \sum x_{i}^{3}+\sum x_{i} \sum y_{i}^{2}-N \sum x_{i} y_{i}^{2} \\
& c_{2}=\sum x_{i}^{2} \sum y_{i}-N \sum y_{i}^{3}+\sum y_{i} \sum y_{i}^{2}-N \sum x_{i}^{2} y_{i} \\
& x_{c}=\frac{c_{1} b_{2}-c_{2} b_{1}}{a_{1} b_{2}-a_{2} b_{1}} \quad y_{c}=\frac{a_{1} c_{2}-a_{2} c_{1}}{a_{1} b_{2}-a_{2} b_{1}} \\
& R^{2}=\frac{1}{N}\left(\sum x_{i}^{2}-2 x_{c} \sum x_{i}+N x_{c}^{2}+\sum y_{i}^{2}-2 y_{c} \sum y_{i}+N y_{c}^{2}\right)
\end{aligned}
$$




\section{Result and Discussion}

Figure 1 is an original coal ash image which the size is $705 \times 503$ pixel with 255 gray-level. The image was processed by median filter to remove the isolated noise points. Smoothing and sharpening processes are performed to enhance the edges before applying Canny's method. In Figure 2, we detect circles by using the procedures described above in the original image. Figure 3 shows the particles. The length of radius drawn in the figure is in logarithmic scale. From these figures, we can see that many circles have been detected correctly with very high accuracy. They are recovered in the real boundaries of particles in the image. However, there are about $20 \%$ particles are failed in the detection. These particles are usually either too small particles or particles with small values of image brightness (i.e. dark particles). Another spurious particles are due to the clustering algorithm. It seems to be very difficult to select a good distance function and its tolerance when clustering, especially for the image like Figure 1, particles vary a lot in their sizes (the largest radius is over 30 pixel, the smallest radius is 3 ). For those small particles, perhaps we should remove them at first by a template method, and then deal with the remains.

\section{References}

1. Canny J, A computational approach to edge detection, Transaction on Pattern Analysis and Machine Intelligence, Vol. PAMI-8, No. 6, pp. 679-698, 1986

2. Castelow DA; Murray DW; Scott GL and Buxton BF, Matching Canny edges to compute the principal components of optic flow, Image and Vision Computing, pp. 129-136, 1988

3. Chen JM; Ventura JA and $\mathrm{Wu} \mathrm{CH}$, Segmentation of planar curves into circular arcs and line segments, Image and Vision Computing, Vol. 14, pp. 71-83, 1996

4. Duda RO and Hart PE, Use of the Hough transformation to detect lines and curves in pictures, Commun. ACM, Vol. 15, pp. 204-208, 1972

5. Lim KB; Xin K; Hong GS, Detection and estimation of circular arc segments, Pattern Recognition Letters, Vol. 16, pp. 627-636, 1995

6. Rosin PL; West GAW, Nonparametric segmentation of curves into various representations, IEEE Transactions on Pattern Analysis and Machine Intelligence, Vol. 17, No. 12, 1995

7. Pla F, Recognition of particle circular shapes from segmented contours, Computer Vision and Image Understanding, Vol. 63, No. 2, pp. 334-343, 1996

8. Thomas SM; Chan YT, A simple approach for the estimation of circular arc center and its radius, Computer Vision, Graphics and Image Processing, Vol. 45, pp. 362-370, 1989

9. 坂上勝彦, 高木幹雄, 反復演算による重なり合った粒子像の分離, 情報処理学会論文誌, Vol. 24, No. 5, 1983

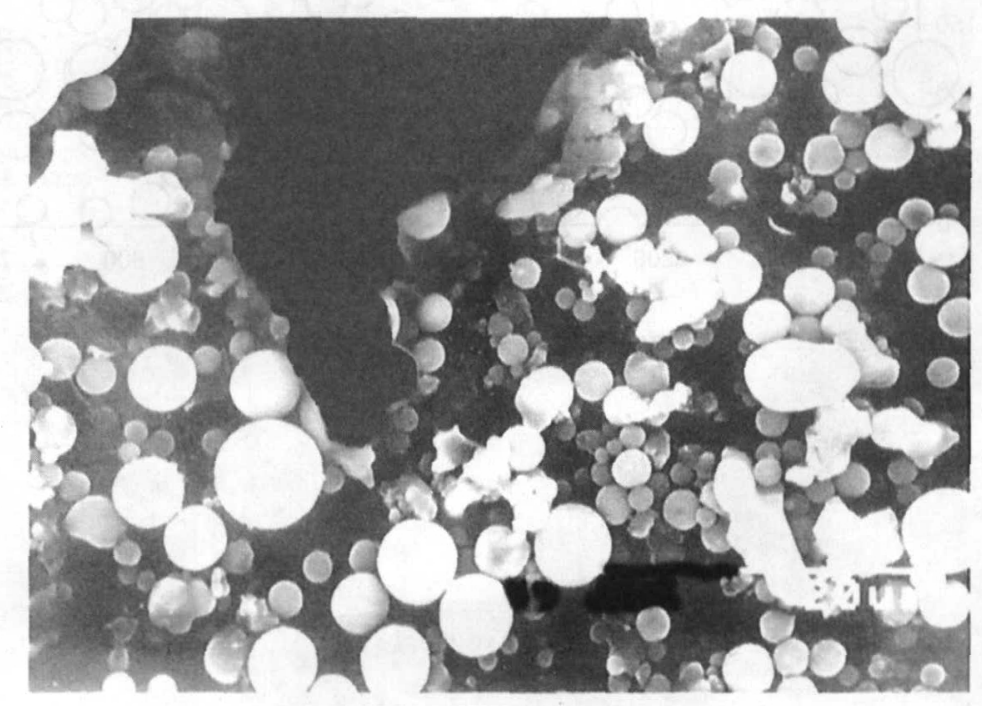

Figure 1: Original image 


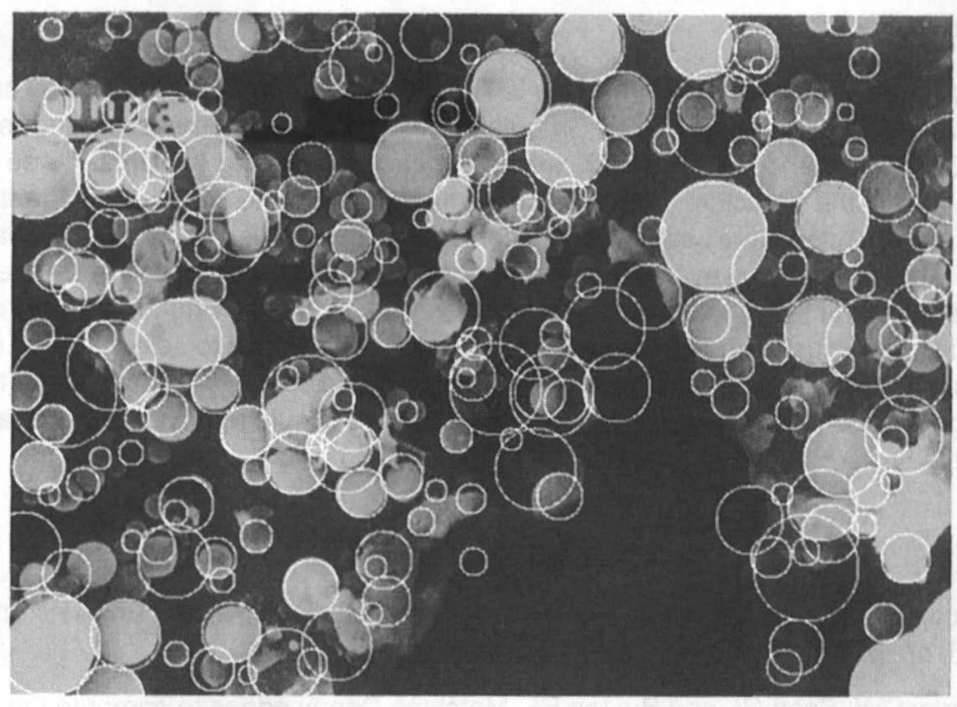

Figure 2: The white circles are drawn on the original image

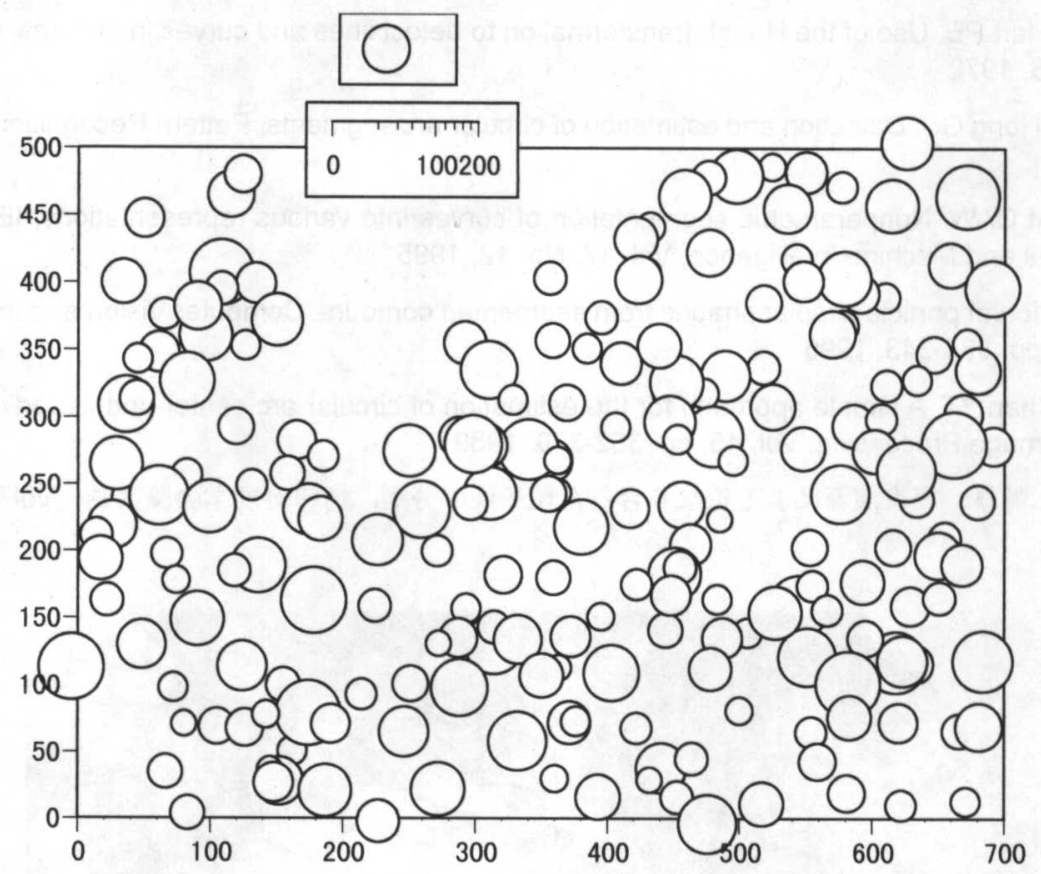

Figure 3: Recovered circles 